\title{
A FIRST-ORDER THEORY OF ULM TYPE
}

\author{
MATTHEW HARRISON-TRAINOR
}

\begin{abstract}
The class of abelian $p$-groups are an example of some very interesting phenomena in computable structure theory. We will give an elementary first-order theory $T_{p}$ whose models are each bi-interpretable with the disjoint union of an abelian $p$-group and a pure set (and so that every abelian $p$-group is bi-interpretable with a model of $T_{p}$ ) using computable infinitary formulas. This answers a question of Knight by giving an example of an elementary firstorder theory of "Ulm type": Any two models, low for $\omega_{1}^{C K}$, and with the same computable infinitary theory, are isomorphic. It also gives a new example of an elementary first-order theory whose isomorphism problem is $\boldsymbol{\Sigma}_{1}^{1}$-complete but not Borel complete.
\end{abstract}

\section{INTRODUCTION}

The class of abelian $p$-groups is a well-studied example in computable structure theory. A simple compactness argument shows that abelian $p$-groups are not axiomatizable by an elementary first-order theory, but they are definable by the conjunction of the axioms for abelian $p$-groups (which are first-order $\forall \exists$ sentences) and the infinitary $\Pi_{2}^{0}$ sentence which says that every element is torsion of order some power of $p$.

Abelian $p$-groups are classifiable by their Ulm sequences Ulm33. Due to this classification, abelian $p$-groups are examples of some very interesting phenomena in computable structure theory and descriptive set theory. We will define a theory $T_{p}$ whose models behave like the class of abelian $p$-groups, giving a first-order example of these phenomena. In particular, Theorem 1.6 below answers a question of Knight.

1.1. Infinitary Formulas. The infinitary logic $\mathcal{L}_{\omega_{1} \omega}$ is the logic which allows countably infinite conjunctions and disjunctions but only finite quantification. If the conjunctions and disjunctions of a formula $\varphi$ are all over computable sets of indices for formulas, then we say that $\varphi$ is computable. We use $\Sigma_{\alpha}^{\text {in }}$ and $\Pi_{\alpha}^{\text {in }}$ to denote the classes of all infinitary $\Sigma_{\alpha}$ and $\Pi_{\alpha}$ formulas respectively. We will also use $\Sigma_{\alpha}^{\mathrm{c}}$ and $\Pi_{\alpha}^{\mathrm{c}}$ to denote the classes of computable $\Sigma_{\alpha}$ and $\Pi_{\alpha}$ formulas, where $\alpha<\omega_{1}^{C K}$ the least non-computable ordinal. See Chapter 6 of [AK00] for a more complete description of computable formulas.

1.2. Bi-Interpretability. One way in which we will see that the models of $T_{p}$ are essentially the same as abelian $p$-group is using bi-interpretations using infinitary formulas [Mon, HTMMM, HTMM]. A structure $\mathcal{A}$ is infinitary interpretable in a structure $\mathcal{B}$ if there is an interpretation of $\mathcal{A}$ in $\mathcal{B}$ where the domain of the interpretation is allowed to be a subset of $\mathcal{B}^{<\omega}$ and where all of the sets in the interpretation are definable using infinitary formulas. This differs from the classical notion of interpretation, as in model theory [Mar02, Definition 1.3.9], where the 
domain is required to be a subset of $\mathcal{B}^{n}$ for some $n$, and the sets in the interpretation are first-order definable.

Definition 1.1. We say that a structure $\mathcal{A}=\left(A ; P_{0}^{\mathcal{A}}, P_{1}^{\mathcal{A}}, \ldots\right)$ (where $\left.P_{i}^{\mathcal{A}} \subseteq A^{a(i)}\right)$ is infinitary interpretable in $\mathcal{B}$ if there exists a sequence of relations $\left(\mathcal{D o m}_{\mathcal{A}}^{\mathcal{B}}\right.$, , $R_{0}, R_{1}, \ldots$ ), definable using infinitary formulas (in the language of $\mathcal{B}$, without parameters), such that

(1) $\mathcal{D o m}_{\mathcal{A}}^{\mathcal{B}} \subseteq \mathcal{B}^{<\omega}$,

(2) $\sim$ is an equivalence relation on $\operatorname{Dom}_{\mathcal{A}}^{\mathcal{B}}$,

(3) $R_{i} \subseteq\left(B^{<\omega}\right)^{a(i)}$ is closed under $\sim$ within $\operatorname{Dom}_{\mathcal{A}}^{\mathcal{B}}$,

and there exists a function $f_{\mathcal{A}}^{\mathcal{B}}: \mathcal{D}$ om ${ }_{\mathcal{A}}^{\mathcal{B}} \rightarrow \mathcal{A}$ which induces an isomorphism:

$$
\left(\mathcal{D o m}_{\mathcal{A}}^{\mathcal{B}} / \sim ; R_{0} / \sim, R_{1} / \sim, \ldots\right) \cong\left(A ; P_{0}^{\mathcal{A}}, P_{1}^{\mathcal{A}}, \ldots\right),
$$

where $R_{i} / \sim$ stands for the $\sim$-collapse of $R_{i}$.

Two structures $\mathcal{A}$ and $\mathcal{B}$ are infinitary bi-interpretable if they are each effectively interpretable in the other, and moreover, the composition of the interpretationsi.e., the isomorphisms which map $\mathcal{A}$ to the copy of $\mathcal{A}$ inside the copy of $\mathcal{B}$ inside $\mathcal{A}$, and $\mathcal{B}$ to the copy of $\mathcal{B}$ inside the copy of $\mathcal{A}$ inside $\mathcal{B}$ - are definable.

Definition 1.2. Two structures $\mathcal{A}$ and $\mathcal{B}$ are infinitary bi-interpretable if there are infinitary interpretations of each structure in the other as in Definition 1.1 such that the compositions

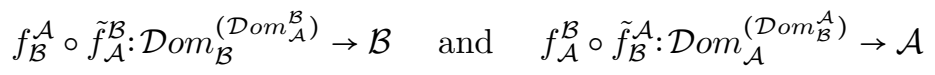

are definable in $\mathcal{B}$ and $\mathcal{A}$ respectively. (Here, we have $\mathcal{D} m_{\mathcal{B}}^{\left(\mathcal{D}^{\prime} m_{\mathcal{A}}^{\mathcal{B}}\right)} \subseteq\left(\mathcal{D} \text { om } m_{\mathcal{A}}^{\mathcal{B}}\right)^{<\omega}$, and $\tilde{f}_{\mathcal{A}}^{\mathcal{B}}:\left(\mathcal{D o m}_{\mathcal{A}}^{\mathcal{B}}\right)^{<\omega} \rightarrow \mathcal{A}^{<\omega}$ is the obvious extension of $f_{\mathcal{A}}^{\mathcal{B}}: \mathcal{D}_{\mathcal{B}} m_{\mathcal{A}}^{\mathcal{B}} \rightarrow \mathcal{A}$ mapping $\operatorname{Dom}_{\mathcal{B}}^{\left(\mathcal{D o m}_{\mathcal{A}}^{\mathcal{B}}\right)}$ to $\operatorname{Dom}_{\mathcal{B}}^{\mathcal{A}}$.)

If we ask that the sets and relations in the interpretation (or bi-interpretation) be (uniformly) relatively intrinsically computable, i.e., definable by both a $\Sigma_{1}^{\mathrm{c}}$ formula and a $\Pi_{1}^{c}$ formula, then we say that the interpretation (or bi-interpretation) is effective. Any two structures which are effectively bi-interpretable have all of the same computability-theoretic properties; for example, they have the same degree spectra and the same Scott rank. See [Mon, Lemma 5.3].

Here, we will use interpretations which use (lightface) $\Delta_{2}^{c}$ formulas. It is no longer true that any two structures which are $\Delta_{2}^{c}$-bi-interpretable have all of the same computability-theoretic properties, but it is true, for example, that any two such structures either both have computable, or both have non-computable, Scott rank.

Theorem 1.3. Each abelian p-group is effectively bi-interpretable with a model of $T_{p}$. Each model of $T_{p}$ is $\Delta_{2}^{c}$-bi-interpretable with the disjoint union of an abelian p-group and a pure set.

This theorem will follow from the constructions in Sections 3 and 4 Given a model $\mathcal{M}$ of $T_{p}, \mathcal{M}$ is bi-interpretable with an abelian $p$-group $G$ and a pure set. The domain of the copy of $G$ inside of $\mathcal{M}$ is definable by a $\Sigma_{1}^{c}$ formula but not by a $\Pi_{1}^{c}$ formula. This is the only part of the bi-interpretation which is not effective. 
1.3. Classification via Ulm Sequences. Let $G$ be an abelian group. For any ordinal $\alpha$, we can define $p^{\alpha} G$ by transfinite induction:

- $p^{0} G=G$;

- $p^{\alpha+1} G=p\left(p^{\alpha} G\right)$;

- $p^{\beta} G=\bigcap_{\alpha<\beta} p^{\alpha} G$ if $\beta$ is a limit ordinal.

These subgroups $p^{\alpha} G$ form a filtration of $G$. This filtration stabilizes, and we call the smallest ordinal $\alpha$ such that $p^{\alpha} G=p^{\alpha+1} G$ the length of $G$. We call the intersection $p^{\infty} G$ of these subgroups, which is a $p$-divisible group, the $p$-divisible part of $G$. Any countable $p$-divisible group is isomorphic to some direct product of the Prüfer group

$$
\mathbb{Z}\left(p^{\infty}\right)=\mathbb{Z}\left[1 / p, 1 / p^{2}, 1 / p^{3}, \ldots\right] / \mathbb{Z} .
$$

Denote by $G[p]$ the subgroup of $G$ consisting of the $p$-torsion elements. The $\alpha$ th Ulm invariant $u_{\alpha}(G)$ of $G$ is the dimension of the quotient

$$
\left(p^{\alpha} G\right)[p] /\left(p^{\alpha+1} G\right)[p]
$$

as a vector space over $\mathbb{Z} / p \mathbb{Z}$.

Theorem 1.4 (Ulm's Theorem, see [Fuc70]). Let $G$ and $H$ be countable abelian $p$-groups such that for every ordinal $\alpha$ their $\alpha$ th Ulm invariants are equal, and the p-divisible parts of $G$ and $H$ are isomorphic. Then $G$ and $H$ are isomorphic.

1.4. Scott Rank and Computable Infinitary Theories. Scott [Sco65] showed that if $\mathcal{M}$ is a countable structure, then there is a sentence $\varphi$ of $\mathcal{L}_{\omega_{1} \omega}$ such that $\mathcal{M}$ is, up to isomorphism, the only countable model of $\varphi$. We call such a sentence a Scott sentence for $\mathcal{M}$. There are many different definitions AK00, Sections 6.6 and 6.7] of the Scott rank of $\mathcal{M}$, which differ only slightly in the ranks they assign. The one we will use, which comes from [Mon15, defines the Scott rank of $\mathcal{A}$ to be the least ordinal $\alpha$ such that $\mathcal{A}$ has a $\Pi_{\alpha+1}^{\text {in }}$ Scott sentence. We denote the Scott rank of a structure $\mathcal{A}$ by $\operatorname{SR}(\mathcal{A})$. It is always the case that $\operatorname{SR}(\mathcal{A}) \leq \omega_{1}^{\mathcal{A}}+1$ Nad74. We could just as easily use any of the other definitions of Scott rank; for all of these definitions, given a computable structure $\mathcal{A}$ :

(1) $\mathcal{A}$ has computable Scott rank if and only if there is a computable ordinal $\alpha$ such that for all tuples $\bar{a}$ in $\mathcal{A}$, the orbit of $\bar{a}$ is defined by a computable $\Sigma_{\alpha}$ formula.

(2) $\mathcal{A}$ has Scott rank $\omega_{1}^{C K}$ if and only if for each tuple $\bar{a}$, the orbit is defined by a computable infinitary formula, but for each computable ordinal $\alpha$, there is a tuple $\bar{a}$ whose orbit is not defined by a computable $\Sigma_{\alpha}$ formula.

(3) $\mathcal{A}$ has Scott rank $\omega_{1}^{C K}+1$ if and only if there is a tuple $\bar{a}$ whose orbit is not defined by a computable infinitary formula.

Given a structure $\mathcal{M}$, define the computable infinitary theory of $\mathcal{M}, \operatorname{Th}_{\infty}(\mathcal{M})$, to be collection of computable $\mathcal{L}_{\omega_{1} \omega}$ sentences true of $\mathcal{M}$. We can ask, for a given structure $\mathcal{M}$, whether $\operatorname{Th}_{\infty}(\mathcal{M})$ is $\aleph_{0}$-categorical, or whether there are other countable models of $\operatorname{Th}_{\infty}(\mathcal{M})$. For $\mathcal{M}$ a hyperarithmetic structure:

(1) If $\operatorname{SR}(\mathcal{M})<\omega_{1}^{C K}$, then $\operatorname{Th}_{\infty}(\mathcal{M})$ is $\aleph_{0}$-categorical. Indeed, $\mathcal{M}$ has a computable Scott sentence $\mathrm{Nad74}$.

(2) If $\operatorname{SR}(\mathcal{M})=\omega_{1}^{C K}$, then $\operatorname{Th}_{\infty}(\mathcal{M})$ may or may not be $\aleph_{0}$-categorical HTIK].

(3) If $\operatorname{SR}(\mathcal{M})=\omega_{1}^{C K}+1$, then $\operatorname{Th}_{\infty}(\mathcal{M})$ is not $\aleph_{0}$-categorical as $\mathcal{M}$ has a non-principal type which may be omitted. 
In the case of abelian $p$-groups, we can say something even when we replace the assumption that $\mathcal{M}$ is hyperarithmetic with the assumption that $\omega_{1}^{G}=\omega_{1}^{C K}$.

Definition 1.5 (Definition 6 of $\left[\mathrm{FKM}^{+} 11\right]$ ). A class of countable structures has Ulm type if for any two structures $\mathcal{A}$ and $\mathcal{B}$ in the class, if $\omega_{1}^{\mathcal{A}}=\omega_{1}^{\mathcal{B}}=\omega_{1}^{C K}$ and $\operatorname{Th}_{\infty}(\mathcal{A})=\operatorname{Th}_{\infty}(\mathcal{B})$, then $\mathcal{A}$ and $\mathcal{B}$ are isomorphic.

It is well-known that abelian $p$-groups are of Ulm type; however, we do not know of a good reference with a complete proof, so we will give one in Section 2 . We also note that there are indeed non-hyperarithmetic abelian $p$-groups $G$ with $\operatorname{SR}(G)<\omega_{1}^{C K}$.

Knight asked whether there was a (non-trivial) first-order theory of Ulm type. By a non-trivial example, we mean that the elementary first-order theory should have non-hyperarithmetic models which are low for $\omega_{1}^{C K}$. Our theory $T_{p}$ is such an example.

Theorem 1.6. The models of $T_{p}$ are of Ulm type. Moreover, given $\mathcal{M} \vDash T_{p}$ with $\omega_{1}^{C K}=\omega_{1}^{\mathcal{M}}$ and $\operatorname{SR}(\mathcal{M})<\omega_{1}^{C K}=\omega_{1}^{\mathcal{M}}, \operatorname{Th}_{\infty}(\mathcal{M})$ is $\aleph_{0}$-categorical.

Proof. Let $\mathcal{M}$ be a model of $T_{p}$. Now $\mathcal{M}$ is bi-interpretable, using computable infinitary formulas, with the disjoint union of an abelian $p$-group $G$ and a pure set. Thus $\mathcal{M}$ inherits these properties from $G$ (see Theorem 2.1).

Of course, there will be non-hyperarithmetic models of $T_{p}$ with Scott rank below $\omega_{1}^{C K}$.

1.5. Borel Incompleteness. In their influential paper [FS89], Friedman and Stanley introduced Borel reductions between invariant Borel classes of structures with universe $\omega$ in a countable language. Such classes are of the form $\operatorname{Mod}(\varphi)$, the set of models of $\varphi$ with universe $\omega$, for some $\varphi \in \mathcal{L}_{\omega_{1} \omega}$. A Borel reduction from $\operatorname{Mod}(\varphi)$ to $\operatorname{Mod}(\psi)$ is a $\operatorname{Borel} \operatorname{map} \Phi: \operatorname{Mod}(\varphi) \rightarrow \operatorname{Mod}(\psi)$ such that

$$
\mathcal{M} \cong \mathcal{N} \Longleftrightarrow \Phi(\mathcal{M}) \cong \Phi(\mathcal{N}) .
$$

If such a Borel reduction exists, we say that $\operatorname{Mod}(\varphi)$ is Borel reducible to $\operatorname{Mod}(\psi)$ and write $\varphi \leq_{B} \psi$. If $\varphi \leq_{B} \psi$ and $\psi \leq_{B} \varphi$, then we say that $\operatorname{Mod}(\varphi)$ and $\operatorname{Mod}(\psi)$ are Borel equivalent and write $\varphi \equiv_{B} \psi$. Friedman and Stanley showed that graphs, fields, linear orders, trees, and groups are all Borel equivalent, and form a maximal class under Borel reduction.

If $\operatorname{Mod}(\varphi)$ is Borel complete, then the isomorphism relation on $\operatorname{Mod}(\varphi) \times \operatorname{Mod}(\varphi)$ is $\boldsymbol{\Sigma}_{1}^{1}$-complete. The converse is not true, and the most well-known example is abelian $p$-groups, whose isomorphism relation is $\boldsymbol{\Sigma}_{1}^{1}$-complete but not Borel complete. Until very recently, they were one of the few such examples, and there were no known examples of elementary first-order theories with similar properties. Recently, Laskowski, Rast, and Ulrich [URL] gave an example of a first-order theory which is not Borel complete, but whose isomorphism relation is not Borel. Our theory $T_{p}$ is another such example.

Theorem 1.7. The class of models of $T_{p}$ is Borel equivalent to abelian p-groups.

Because abelian $p$-groups are not Borel complete, but their isomorphism relation is $\boldsymbol{\Sigma}_{1}^{1}$-complete, we get:

Corollary 1.8. The class of models of $T_{p}$ is not Borel complete but the isomorphism relation is $\boldsymbol{\Sigma}_{1}^{1}$-complete. 
Theorem 1.7 is a specific instance of the following general question asked by Friedman:

Question 1.9. Is it true that for every $\mathcal{L}_{\omega_{1} \omega}$ sentence there is a Borel equivalent first-order theory?

\section{Abelian $p$-groups are of Ulm type}

In this section we will describe a proof of the following well-known theorem, which shows that abelian $p$-groups are of Ulm type.

Theorem 2.1. Let $G$ be an abelian p-group with $\omega_{1}^{C K}=\omega_{1}^{G}$. Then:

(1) $G$ is the only countable model of $\mathrm{Th}_{\infty}(G)$ with $\omega_{1}^{G}=\omega_{1}^{C K}$, and

(2) if $\operatorname{SR}(G)<\omega_{1}^{C K}=\omega_{1}^{G}$, then $\operatorname{Th}_{\infty}(G)$ is $\aleph_{0}$-categorical.

The proof of Theorem 2.1 consists essentially of expressing the Ulm invariants via computable infinitary formulas.

Definition 2.2. Let $G$ be an abelian $p$-group. For each ordinal $\alpha<\omega_{1}^{C K}$, there is a computable infinitary sentence $\psi_{\alpha}(x)$ which defines $p^{\alpha} G$ inside of $G$ :

- $\psi_{0}(x)$ is just $x=x$;

- $\psi_{\alpha+1}(x)$ is $(\exists y)\left[\psi_{\alpha}(y) \wedge p y=x\right]$;

- $\psi_{\beta}(x)$ is $\mathbb{A}_{\alpha<\beta} \psi_{\alpha}(x)$ for limit ordinals $\beta$.

Definition 2.3. For each ordinal $\alpha<\omega_{1}^{C K}$ and $n \in \omega \cup\{\omega\}$, there is a computable infinitary sentence $\varphi_{\alpha, n}$ such that, for $G$ an abelian $p$-group,

$$
G \vDash \varphi_{\alpha, n} \Leftrightarrow u_{\alpha}(G)=n .
$$

For $n \in \omega$, define $\varphi_{\alpha, \geq n}$ to say that there are $x_{1}, \ldots, x_{n}$ such that:

- $\psi_{\alpha}\left(x_{1}\right) \wedge \cdots \wedge \psi_{\alpha}\left(x_{n}\right)$,

- $p x_{1}=\cdots=p x_{n}=0$, and

- for all $c_{1}, \ldots, c_{n} \in \mathbb{Z} / p \mathbb{Z}$ not all zero, $\neg \psi_{\alpha+1}\left(c_{1} x_{1}+\cdots+c_{n} x_{n}\right)$.

Then for $n \in \omega, \varphi_{\alpha, n}$ is $\varphi_{\alpha, \geq n} \wedge \neg \varphi_{\alpha, \geq n+1}$, and $\varphi_{\alpha, \omega}$ is $\mathbb{A}_{n \in \omega} \varphi_{\alpha, \geq n}$.

Lemma 2.4 (Theorem 8.17 of [AK00]). Let $G$ be an abelian p-group. Then:

(1) the length of $G$ is at most $\omega_{1}^{G}$, and

(2) if $G$ has length $\omega_{1}^{G}$ then $G$ is not reduced (in fact, its p-divisible part has infinite rank) and $\operatorname{SR}(G)=\omega_{1}^{G}+1$.

We are now ready to give the proof of Theorem 2.1 .

Proof of Theorem 2.1. Since $\omega_{1}^{C K}=\omega_{1}^{G}, G$ has length at most $\omega_{1}^{C K}$. Note that $\mathrm{Th}_{\infty}(G)$ contains the sentences $\varphi_{\alpha, u_{\alpha}(G)}$ for $\alpha<\omega_{1}^{C K}$. Thus any model of $\mathrm{Th}_{\infty}(G)$ has the same Ulm invariants as $G$, for ordinals below $\omega_{1}^{C K}$.

If $\operatorname{SR}(G)<\omega_{1}^{C K}$, let $\lambda$ be the length of $G$. Then $\operatorname{Th}_{\infty}(G)$ includes the computable formula $(\forall x)\left[\psi_{\lambda}(x) \leftrightarrow \psi_{\lambda+1}(x)\right]$, so that any countable model of $\operatorname{Th}_{\infty}(G)$ has length at most $\lambda$. Note that in such a model, $\psi_{\lambda}$ defines the $p$-divisible part. Let $n \in \omega \cup\{\omega\}$ be such that $p^{\infty} G$ is isomorphic to $\mathbb{Z}\left(p^{\infty}\right)^{n}$. Then, if $n \in \omega, \mathrm{Th}_{\infty}(G)$ contains the formula which says that there are $x_{1}, \ldots, x_{n}$ such that

- $\psi_{\lambda}\left(x_{1}\right) \wedge \cdots \wedge \psi_{\lambda}\left(x_{n}\right)$,

- for all $c_{1}, \ldots, c_{n}<p$ not all zero and $k_{1}, \ldots, k_{n} \in \omega$,

$$
\frac{c_{1}}{p^{k_{1}}} x_{1}+\cdots+\frac{c_{n}}{p^{k_{n}}} x_{n} \neq 0,
$$


- for all $y$ with $\psi_{\lambda}(y)$, there are $c_{1}, \ldots, c_{n}<p$ and $k_{1}, \ldots, k_{n} \in \omega$ such that

$$
y=\frac{c_{1}}{p^{k_{1}}} x_{1}+\cdots+\frac{c_{n}}{p^{k_{n}}} x_{n} .
$$

If $n=\omega$, then $\operatorname{Th}_{\infty}(G)$ contains the formula which says that for each $m \in \omega$, there are $x_{1}, \ldots, x_{m}$ such that

- $\psi_{\lambda}\left(x_{1}\right) \wedge \cdots \wedge \psi_{\lambda}\left(x_{m}\right)$, and

- for all $c_{1}, \ldots, c_{m}<p$ not all zero and $k_{1}, \ldots, k_{m} \in \omega$,

$$
\frac{c_{1}}{p^{k_{1}}} x_{1}+\cdots+\frac{c_{m}}{p^{k_{m}}} x_{m} \neq 0 .
$$

Any countable model of $\operatorname{Th}_{\infty}(G)$ has $p$-divisible part isomorphic to $\mathbb{Z}\left(p^{\infty}\right)^{n}$. So any countable model of $\operatorname{Th}_{\infty}(G)$ has the same Ulm invariants and $p$-divisible part as $G$, and hence is isomorphic to $\operatorname{Th}_{\infty}(G)$. Hence $\operatorname{Th}_{\infty}(G)$ is $\aleph_{0}$-categorical. This gives (2), and (1) for the case where $\operatorname{SR}(G)<\omega_{1}^{C K}$.

If $\operatorname{SR}(G)=\omega_{1}^{C K}+1$, let $H$ be any other countable model of $\operatorname{Th}_{\infty}(G)$ with $\omega_{1}^{H}=\omega_{1}^{G}=\omega_{1}^{C K}$. Thus $G$ and $H$ both have length $\omega_{1}^{C K}$ and their $p$-divisible parts have infinite rank. As remarked before, they have the same Ulm invariants, and so they must be isomorphic. This completes the proof of (1).

\section{The Theory $T_{p}$}

Fix a prime $p$. The language $\mathcal{L}_{p}$ of $T_{p}$ will consist of a constant 0 , unary relations $R_{n}$ for $n \in \omega$, and ternary relations $P_{\ell, m}^{n}$ for $\ell, m \in \omega$ and $n \leq \max (\ell, m)$. The following transformation of an abelian $p$-group into an $\mathcal{L}_{p}$-structure will illustrate the intended meaning of the symbols.

Definition 3.1. Let $G$ be an abelian $p$-group. Define $\mathfrak{M}(G)$ to be $\mathcal{L}_{p}$-structure obtained as follows, with the same domain as $G$, and the symbols of $\mathcal{L}_{p}$ interpreted as follows:

- Set $0^{\mathfrak{M}(G)}$ to be the identity element of $G$.

- For each $n$, let $R_{n}^{\mathfrak{M}(G)}$ be the elements which are torsion of order $p^{n}$.

- For each $\ell, m \in \omega$ and $n \leq \max (\ell, m)$, and $x, y, z \in G$, set $P_{\ell, m}^{n, \mathfrak{M}(G)}(x, y, z)$ if and only if $x+y=z, x \in R_{\ell}^{\mathfrak{M}(G)}, y \in R_{m}^{\mathfrak{M}(G)}$, and $z \in R_{n}^{\mathfrak{M}(G)}$.

One should think of such $\mathcal{L}_{p}$-structures as the canonical models of $T_{p}$. The theory $T_{p}$ will consist of following axiom schemata:

(A1) For all $\ell, m, n \in \omega$ :

$$
(\forall x \forall y \forall z)\left[P_{\ell, m}^{n}(x, y, z) \rightarrow\left(R_{\ell}(x) \wedge R_{m}(x) \wedge R_{n}(z)\right)\right] .
$$

(A2) ( $R_{n}$ contains the elements which are torsion of order $p^{n}$.)

$$
(\forall x)\left[R_{0}(x) \leftrightarrow x=0\right] .
$$

and, for all $n \geq 1$ :

$(\forall x)\left[x \in R_{n} \leftrightarrow\left(\exists x_{2} \cdots \exists x_{p-1}\right)\left[P_{n, n}^{n}\left(x, x, x_{2}\right) \wedge P_{n, n}^{n}\left(x, x_{2}, x_{3}\right) \wedge \cdots \wedge P_{n, n}^{n-1}\left(x, x_{p-1}, x_{p}\right)\right]\right]$.

(A3) (P defines a partial function.) For all $\ell, m, n, n^{\prime} \in \omega$ :

$$
\left(\forall x \forall y \forall z \forall z^{\prime}\right)\left[\left(P_{\ell, m}^{n}(x, y, z) \wedge P_{\ell, m}^{n^{\prime}}\left(x, y, z^{\prime}\right)\right) \rightarrow z=z^{\prime}\right] .
$$


(A4) (P is total.) For all $\ell, m \in \omega$ :

$$
(\forall x \forall y)\left[\left(R_{\ell}(x) \wedge R_{m}(y)\right) \rightarrow \bigvee_{n \leq \max (\ell, m)}(\exists z) P_{\ell, m}^{n}(x, y, z)\right] .
$$

(A5) (Identity.) For all $\ell \in \omega$ :

$$
(\forall x)\left[R_{\ell}(x) \rightarrow\left[P_{0, \ell}^{\ell}(0, x, x) \wedge P_{\ell, 0}^{\ell}(x, 0, x)\right]\right] .
$$

(A6) (Inverses.) For all $\ell \in \omega$ :

$$
(\forall x)(\exists y)\left[R_{\ell}(x) \rightarrow\left[P_{\ell, \ell}^{0}(x, y, 0) \wedge P_{\ell, \ell}^{0}(y, x, 0)\right]\right] .
$$

(A7) (Associativity.) For all $\ell, m, n \in \omega$ :

$$
\begin{aligned}
& (\forall x \forall y \forall z)\left[\left[R_{\ell}(x) \wedge R_{m}(y) \wedge R_{n}(z)\right] \rightarrow\right. \\
& \left.\underset{\substack{r \leq \max (\ell, m) \\
s \leq \max (m, n) \\
t \leq \max (r, n), \max (\ell, s)}}{\bigvee}(\exists u \exists v \exists w)\left[P_{\ell, m}^{r}(x, y, u) \wedge P_{r, n}^{t}(u, z, w) \wedge P_{m, n}^{s}(y, z, v) \wedge P_{\ell, s}^{t}(x, v, w)\right]\right] .
\end{aligned}
$$

(A8) (Abelian.) For all $\ell, m \in \omega$ and $n \leq \max (\ell, m)$ :

$$
(\forall x \forall y \forall z)\left[\left[R_{\ell}(x) \wedge R_{m}(y) \wedge R_{n}(z) \wedge P_{\ell, m}^{n}(x, y, z)\right] \rightarrow P_{m, \ell}^{n}(y, x, z)\right] .
$$

We must now check that the definition of $T_{p}$ works as desired, that is, that if $G$ is an abelian $p$-group, then $\mathfrak{M}(G)$ is a model of $T_{p}$.

Lemma 3.2. If $G$ is an abelian $p$-group, then $\mathfrak{M}(G) \vDash T_{p}$.

Proof. We must check that each instance of the axiom schemata of $T_{p}$ holds in $\mathfrak{M}(G)$.

(A1) Suppose that $x, y$, and $z$ are elements of $G$ with $P_{m, \ell}^{n, \mathfrak{M}(G)}(x, y, z)$. Then, by definition, $x+y=z, x \in R_{\ell}^{\mathfrak{M}}(G), y \in R_{m}^{\mathfrak{M}(G)}$, and $z \in R_{n}^{\mathfrak{M}(G)}$.

(A2) $R_{0}^{\mathfrak{M}(G)}$ contains the elements of $G$ which are torsion of order $p^{0}=1$, so $R_{0}$ contains just the identity. For each $n>0, R_{n}^{\mathfrak{M}(G)}$ contains the elements of order $p^{n}$. An element $x$ has order $p^{n}$ if and only if $p x$ has order $p^{n-1}$. It remains only to note that if $x$ has order $p^{n}$, then $x, 2 x, 3 x, \ldots,(p-1) x$ all have order $p^{n}$ as well. The existential quantifier is witnessed by $x_{2}=2 x$, $x_{3}=3 x$, and so on.

(A3) If, for some $x, y, z$, and $z^{\prime}, P_{\ell, m}^{n, \mathfrak{M}(G)}(x, y, z)$ and $P_{\ell, m}^{n^{\prime}, \mathfrak{M}(G)}\left(x, y, z^{\prime}\right)$, then $x+y=z$ and $x+y=z^{\prime}$, so that $z=z^{\prime}$.

(A4) Given $x$ and $y$ in $G$ which are of order $p^{m}$ and $p^{\ell}$ respectively, $x+y$ is of order $p^{n}$ for some $n \leq \max (m, \ell)$, and so we have $P_{m, \ell}^{n, \mathfrak{M}(G)}(x, y, x+y)$.

(A5) If $x \in G$ is of order $p^{\ell}$, then $x+0=0+x=x$ and so we have $P_{\ell, 0}^{\ell, \mathfrak{M}(G)}(x, 0, x)$.

(A6) If $x \in G$ is of order $p^{\ell}$, then $-x$ is also of order $p^{\ell}$, and $x+(-x)=0=(-x)+x$. So we have $P_{\ell, \ell}^{0, \mathfrak{M}(G)}(x,-x, 0)$.

(A7) Given $x, y, z \in G$ of order $p^{\ell}, p^{m}$, and $p^{n}$ respectively, there are $r \leq \max (\ell, m)$ and $s \leq \max (m, n)$ such that $x+y$ and $y+z$ are of order $p^{r}$ and $p^{s}$ respectively. Then there is $t$ such that $x+y+z$ is of order $p^{t} ; t \leq \max (r, n)$ and $t \leq \max (\ell, s)$. 
(A8) Given $x, y, z \in G$ of order $p^{\ell}, p^{m}$, and $p^{n}$ respectively, $n \leq \max (\ell, m)$, and with $x+y=z$, we have $y+x=z$ as $G$ is abelian.

Thus we have shown that $\mathfrak{M}(G)$ is a model of $T_{p}$.

Note that $G$ and $\mathfrak{M}(G)$ are effectively bi-interpretable, proving one half of Theorem 1.3

\section{From a model of $T_{p}$ TO AN ABELIAN $p$-Group}

Given an abelian $p$-group $G$, we have already described how to turn $G$ into a model of $T_{p}$. In this section we will do the reverse by turning a model of $T_{p}$ into an abelian $p$-group.

Definition 4.1. Let $\mathcal{M}$ be a model of $T_{p}$. Define $\mathfrak{G}(\mathcal{M})$ to be the group obtained as follows.

- The domain of $\mathfrak{G}(\mathcal{M})$ will be the subset of the domain of $\mathcal{M}$ given by $\cup_{n \in \omega} R_{n}^{\mathcal{M}}$.

- The identity element of $\mathfrak{G}(\mathcal{M})$ will be $0^{\mathcal{M}}$.

- We will have $x+y=z$ in $\mathfrak{G}(\mathcal{M})$ if and only if, for some $\ell, m$, and $n$, $P_{\ell, m}^{n, \mathcal{M}}(x, y, z)$.

We will now check that $\mathfrak{G}(\mathcal{M})$ is always an abelian $p$-group.

Lemma 4.2. If $\mathcal{M}$ is a model of $T_{p}$, then $\mathfrak{G}(\mathcal{M})$ is an abelian p-group.

Proof. First we check that the operation + on $\mathfrak{G}(\mathcal{M})$ defines a total function. Given $x, y \in \mathfrak{G}(\mathcal{M})$, choose $\ell$ and $m$ such that $x \in R_{\ell}^{\mathcal{M}}$ and $y \in R_{m}^{\mathcal{M}}$. Then by (A3) and (A4), there is a unique $n \leq \max (\ell, m)$ and a unique $z$ such that $P_{\ell, m}^{n, \mathcal{M}}(x, y, z)$. Thus $x+y=z$, and $z$ is unique.

Second, we check that $\mathfrak{G}(\mathcal{M})$ is in fact a group. To see that $0^{\mathcal{M}}$ is the identity, given $x \in \mathfrak{G}(\mathcal{M})$, there is $\ell$ such that $x \in R_{\ell}^{\mathcal{M}}$. By $(\mathrm{A} 5), P_{\ell, 0}^{\ell, \mathcal{M}}\left(x, 0^{\mathcal{M}}, x\right)$ and $P_{0, \ell}^{\ell, \mathcal{M}}\left(0^{\mathcal{M}}, x, 0^{\mathcal{M}}\right)$. Thus $x+0^{\mathcal{M}}=0^{\mathcal{M}}+x=x$, and $0^{\mathcal{M}}$ is the identity of $\mathfrak{G}(\mathcal{M})$. To see that $\mathfrak{G}(\mathcal{M})$ has inverses, given $x \in \mathfrak{G}(\mathcal{M})$, there is $\ell$ such that $x \in R_{\ell}^{\mathcal{M}}$, and by (A6) there is $y \in R_{\ell}^{\mathcal{M}}$ such that $P_{\ell, \ell}^{0, \mathcal{M}}\left(x, y, 0^{\mathcal{M}}\right)$ and $P_{\ell, \ell}^{0, \mathcal{M}}\left(y, x, 0^{\mathcal{M}}\right)$. Thus $x+y=y+x=0^{\mathcal{M}}$, and so $y$ is the inverse of $x$. Finally, to see that $\mathfrak{G}(M)$ is associative, given $x, y, z \in \mathfrak{G}(\mathcal{M})$, there are $\ell, m$, and $n$ such that $x \in R_{\ell}^{\mathcal{M}}, y \in R_{m}^{\mathcal{M}}$, and $z \in R_{n}^{\mathcal{M}}$. Then by (A7) there are $r, s$, and $t$, and $u, v$, and $w$, such that $P_{\ell, m}^{r, \mathcal{M}}(x, y, u), P_{r, n}^{t, \mathcal{M}}(u, z, w), P_{m, n}^{s, \mathcal{M}}(y, z, v)$, and $P_{\ell, s}^{t, \mathcal{M}}(x, v, w)$. Thus $x+y=u$, $u+z=w, y+z=v$, and $x+v=w$. So $(x+y)+z=x+(y+z)$. Thus $\mathfrak{G}(\mathcal{M})$ is associative.

Third, to see that $\mathfrak{G}(\mathcal{M})$ is abelian, let $x, y \in \mathfrak{G}(\mathcal{M})$. There are $\ell$ and $m$ such that $x \in R_{\ell}^{\mathcal{M}}$ and $y \in R_{m}^{\mathcal{M}}$. Let $n \leq \max (\ell, m)$ be such that $z=x+y \in R_{n}^{\mathcal{M}}$. (Such an $n$ and $z$ exist by the arguments above that + is total, via (A3) and (A4).) Then $P_{\ell, m}^{n, \mathcal{M}}(x, y, z)$, and so by $(\mathrm{A} 8), P_{m, \ell}^{n, \mathcal{M}}(y, x, z)$. Thus $y+x=z$ and so $\mathfrak{G}(\mathcal{M})$ is abelian.

Finally, we need to see that $\mathfrak{G}(\mathcal{M})$ is a $p$-group. We claim, by induction on $n \geq 0$, that $R_{n}^{\mathcal{M}}$ consists of the elements of $\mathfrak{G}(\mathcal{M})$ which are of order $p^{n}$. From this claim, it follows that $\mathfrak{G}(\mathcal{M})$ is a $p$-group. For $n=0$, the claim follows directly from (A2). Given $n>0$, suppose that $x \in R_{n}^{\mathcal{M}}$. Then the witnesses $x_{2}, x_{3}, \ldots, x_{p}$ to (A2) must be $2 x, 3 x, \ldots, p x$. Note that since $P_{n, n}^{n-1, \mathcal{M}}(x,(p-1) x, p x), p x \in R_{n-1}^{\mathcal{M}}$. Thus $p x$ is of 
order $p^{n-1}$, and so $x$ is of order $p^{n}$. On the other hand, if $x$ is of order $p^{n}$, then $p x$ is of order $p^{n-1}$ and so $p x \in R_{n-1}^{\mathcal{M}}$. Moreover, $x_{2}=2 x, x_{3}=3 x, \ldots, x_{p-1}=(p-1) x$ are all of order $p^{n}$. So we have $P_{n, n}^{n, \mathcal{M}}\left(x, x, x_{2}\right), P_{n, n}^{n, \mathcal{M}}\left(x, x_{2}, x_{3}\right), \ldots, P_{n, n}^{n-1, \mathcal{M}}\left(x, x_{p-1}, x_{p}\right)$. By (A2), $x \in R_{n}^{\mathcal{M}}$. This completes the inductive proof.

We now have two operations, one which turns an abelian $p$-group into a model of $T_{p}$, and another which turns a model of $T_{p}$ into an abelian $p$-group. These two operations are almost inverses to each other. If we begin with an abelian $p$-group, turn it into a model of $T_{p}$, and then that model into an abelian $p$-group, we will obtain the original group. However, if we start with a $\mathcal{M}$ model of $T_{p}$, turn it into an abelian $p$-group, and then turn that abelian $p$-group into a model of $T_{p}$, we may obtain a different model of $T_{p}$. The problem is that the of elements of $\mathcal{M}$ which are not in any of the sets $R_{n}^{\mathcal{M}}$ are discarded when we transform $\mathcal{M}$ into an abelian $p$-group. However, these elements form a pure set, and so the only pertinent information is their size.

Definition 4.3. Given a model $\mathcal{M}$ of $T_{p}$, the size of $\mathcal{M}, \# \mathcal{M} \in \omega \cup\{\infty\}$, is the number of elements of $M$ not in any relation $R_{n}$.

Lemma 4.4. Given an abelian p-group $G, \mathfrak{G}(\mathfrak{M}(G))=G$.

Proof. Since $\# \mathfrak{M}(G)=0$, we see that $G, \mathfrak{M}(G)$, and $\mathfrak{G}(\mathfrak{M}(G))$ all have the same domain. The identity of $\mathfrak{G}(\mathfrak{M}(G))$ is $0^{\mathfrak{M}(G)}$ which is the identity of $G$. If $x+y=z$ in $G$, then, for some $\ell, m, n \in \omega$, we have $P_{\ell, m}^{n, \mathfrak{M}(G)}(x, y, z)$. Thus, in $\mathfrak{G}(\mathfrak{M}(G))$, we have $x+y=z$. So $\mathfrak{G}(\mathfrak{M}(G))=G$.

We make a simple extension to $\mathfrak{M}$ as follows.

Definition 4.5. Let $G$ be an abelian $p$-group and $m \in \omega \cup\{\infty\}$. Define $\mathfrak{M}(G, m)$ to be $\mathcal{L}_{p}$-structure with domain $G \cup\left\{a_{1}, \ldots, a_{m}\right\}$ with the relations interpreted as in $\mathfrak{M}(G)$. Thus, no relations hold of any of the elements $a_{1}, \ldots, a_{m}$.

Lemma 4.6. Given a model $\mathcal{M}$ of $T_{p}, \mathfrak{M}(G(\mathcal{M}), \# \mathcal{M}) \cong \mathcal{M}$.

Proof. We will show that if $\# \mathcal{M}=0$, then $\mathfrak{M}(\mathfrak{G}(\mathcal{M}))=\mathcal{M}$. From this one can easily see that $\mathfrak{M}(G(\mathcal{M}), \# \mathcal{M}) \cong \mathcal{M}$ in general.

If $\# \mathcal{M}=0$, then $\mathcal{M}, \mathfrak{G}(\mathcal{M})$, and $\mathfrak{M}(\mathfrak{G}(\mathcal{M}))$ all share the same domain. It is clear that $0^{\mathcal{M}}=0^{\mathfrak{G}(\mathcal{M})}=0^{\mathfrak{M}(\mathfrak{G}(\mathcal{M}))}$. From the proof of Lemma 4.2, we see that for each $n, R_{n}^{\mathcal{M}}$ defines the set of elements of $\mathfrak{G}(\mathcal{M})$ which are torsion of order $p^{n}$, and so $R_{n}^{\mathcal{M}}=R_{n}^{\mathfrak{M}(\mathfrak{G}(\mathcal{M}))}$. Given $\ell, m \in \omega$ and $n \leq \max (\ell, m)$, and $x, y$, and $z$ elements of the shared domain, we have $P_{\ell, m}^{n, \mathcal{M}}(x, y, z)$ if and only if

$$
x+y=z \text { in } \mathfrak{G}(\mathcal{M}) \text { and } x \in R_{\ell}^{\mathcal{M}}, y \in R_{m}^{\mathcal{M}} \text {, and } z \in R_{n}^{\mathcal{M}} .
$$

Since $R_{i}^{\mathcal{M}}=R_{i}^{\mathfrak{M}(\mathfrak{G}(\mathcal{M}))}$ for each $i$, this is the case if and only if $P_{\ell, m}^{n, \mathfrak{M}(\mathfrak{G}(\mathcal{M}))}(x, y, z)$. Thus we have shown that $\mathfrak{M}(\mathfrak{G}(\mathcal{M}))=\mathcal{M}$.

Note that $\mathcal{M}$ and the disjoint union of $\mathfrak{G}(\mathcal{M})$ with a pure set of size $\# \mathcal{M}$ are bi-interpretable, using computable infinitary formulas, completing the proof of Theorem 1.3 . 


\section{Borel Equivalence}

In this section we will prove Theorem 1.7 by showing that the class of models of $T_{p}$ and the class of abelian $p$-groups are Borel equivalent. $G \mapsto \mathfrak{G}(\mathfrak{M}(G))=$ $\mathfrak{G}(\mathfrak{M}(G, 0))$ is a Borel reduction from isomorphism on abelian $p$-groups to isomorphism on models of $T_{p}$. However, $\mathcal{M} \mapsto \mathfrak{G}(\mathcal{M})$ is not a Borel reduction in the other direction, because two non-isomorphic models of $T_{p}$ might be mapped to isomorphic groups. We need to find a way to turn $\mathfrak{G}(\mathcal{M})$ and $\# \mathcal{M}$ into an abelian $p$-group

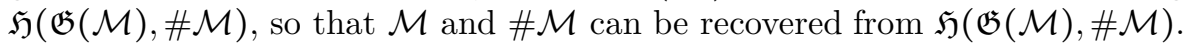

We will define $\mathfrak{H}(G, m)$ for any abelian $p$-group $H$ and $m \in \omega \cup\{\infty\}$. It is helpful to think about what this reduction will do to the Ulm invariants: The first Ulm invariant of $\mathfrak{H}(G, m)$ will be $m$, and for each $\alpha$, then $1+\alpha$ th Ulm invariant of $\mathfrak{H}(G, m)$ will be the same as the $\alpha$ th Ulm invariant of $G$.

Definition 5.1. Given an abelian $p$-group $G$, and $m \in \omega \cup\{\infty\}$, define an abelian $p$ group $\mathfrak{H}(G, m)$ as follows. Let $\hat{\mathcal{B}}$ be a basis for the $\mathbb{Z}_{p}$-vector space $G / p G$. Let $\mathcal{B} \subseteq G$ be a set of representatives for $\hat{\mathcal{B}}$. Let $G^{*}$ be the abelian group $\left\langle G, a_{b}: b \in \mathcal{B} \mid p a_{b}=b\right\rangle$. Then define $\mathfrak{H}(G, m)=G^{*} \oplus\left(\mathbb{Z}_{p}\right)^{m}$.

To make this Borel, we can take $\mathcal{B}$ to be the lexicographically first set of representatives for a basis. It will follow from Lemma 5.4 that the isomorphism type of $\mathfrak{H}(G, m)$ does not depend on these choices. First, we require a couple of lemmas.

Lemma 5.2. Each element of $G$ can be written uniquely as a (finite) linear combination $h+\sum_{b \in \mathcal{B}} x_{b} b$ where $h \in p G$ and each $x_{b}<p$.

Proof. Given $g \in G$, let $\hat{g}$ be the image of $g$ in $G / p G$. Then, since $\hat{\mathcal{B}}$ is a basis for $G / p G$, we can write

$$
\hat{g}=\sum_{b \in \mathcal{B}} x_{b} \hat{b}
$$

with $x_{b}<p$, where $\hat{b}$ is the image of $b$ in $G / p G$. Thus setting

$$
h=g-\sum_{b \in \mathcal{B}} x_{b} b \in p G
$$

we get a representation of $g$ as in the statement of the theorem.

To see that this representation is unique, suppose that

$$
h+\sum_{b \in \mathcal{B}} x_{b} b=h^{\prime}+\sum_{b \in \mathcal{B}} y_{b} b
$$

Then, modulo $p G$,

$$
\sum_{b \in \mathcal{B}} x_{b} \hat{b}=\sum_{b \in \mathcal{B}} y_{b} \hat{b}
$$

Since $\hat{\mathcal{B}}$ is a basis, $x_{b}=y_{b}$ for each $b \in \mathcal{B}$. Then we get that $h=h^{\prime}$ and the two representations are the same.

Lemma 5.3. Each element of $G^{*}$ can be written uniquely in the form $h+\sum_{b \in \mathcal{B}} x_{b} a_{b}$ where $h \in G$ and each $x_{b}<p$.

Proof. It is clear that each element of $G^{*}$ can be written in such a way. If

$$
h+\sum_{b \in \mathcal{B}} x_{b} a_{b}=h^{\prime}+\sum_{b \in \mathcal{B}} y_{b} a_{b}
$$

then, in $G$,

$$
p h+\sum_{b \in \mathcal{B}} x_{b} b=p h^{\prime}+\sum_{b \in \mathcal{B}} y_{b} b .
$$


This representation is unique, so $x_{b}=y_{b}$ for each $b \in \mathcal{B}$, and so $h=h^{\prime}$.

Lemma 5.4. The isomorphism type of $\mathfrak{H}(G, m)$ depends only on the isomorphism type of $G$, and not on the choice of $\mathcal{B}$.

Proof. It suffices to show that if $\mathcal{C}$ is another choice of representatives for a basis of $G / p G$, then $G_{\mathcal{B}}^{*}=G_{\mathcal{C}}^{*}$, where the former is constructed using $\mathcal{B}$, and the later is constructed using $\mathcal{C}$. Let $f: \mathcal{B} \rightarrow \mathcal{C}$ be an bijection.

Given $g \in G_{\mathcal{B}}^{*}$, write $g=g^{\prime}+\sum_{b \in \mathcal{B}} x_{b} a_{b}$ with $g^{\prime} \in G$ and $0 \leq x_{b}<p$. This representation of $g$ is unique by Lemma 5.3. Define $\varphi(g)=g^{\prime}+\sum_{b \in \mathcal{B}} x_{b} a_{f(b)}$. It is not hard to check that $\varphi$ is a homomorphism. The inverse of $\varphi$ is the map $\psi$ which is defined by $\psi(h)=h^{\prime}+\sum_{c \in \mathcal{C}} y_{c} a_{f^{-1}(c)}$ where $h=h^{\prime}+\sum_{c \in \mathcal{C}} y_{c} a_{c}$.

The next two lemmas will be used to show that if $G$ is not isomorphic to $G^{\prime}$, or if $m$ is not equal to $m^{\prime}$, then $\mathfrak{H}(G, m)$ will not be isomorphic to $\mathfrak{H}\left(G^{\prime}, m^{\prime}\right)$.

Lemma 5.5. $G=p G^{*}$.

Proof. Each element of $G$ can be written as $g+\sum_{b \in \mathcal{B}} x_{b} b$ with $g \in p G$. Let $g^{\prime} \in G$ be such that $p g^{\prime}=g$. Then

$$
p\left(g^{\prime}+\sum_{b \in \mathcal{B}} x_{b} a_{b}\right)=g+\sum_{b \in \mathcal{B}} x_{b} b .
$$

Hence $G \subseteq p G^{*}$. Given $h \in G^{*}$, write $h=g+\sum_{b \in \mathcal{B}} x_{b} a_{b}$. Then $p h=p g+\sum_{b \in \mathcal{B}} x_{b} b \in G$. So $p G^{*} \subseteq G$, and so $G=p G^{*}$.

If $G$ is a group, recall that we denote by $G[p]$ the elements of $G$ which are torsion of order $p$.

Lemma 5.6. $\mathfrak{H}(G, m)[p] /(p \mathfrak{H}(G, m))[p] \cong\left(\mathbb{Z}_{p}\right)^{m}$.

Proof. Note that

$$
\begin{aligned}
\mathfrak{H}(G, m)[p] /(p \mathfrak{H}(G, m))[p] & \cong\left(G^{*}[p] /\left(p G^{*}\right)[p]\right) \oplus\left(\left(\mathbb{Z}_{p}\right)^{m}[p] /\left(p\left(\mathbb{Z}_{p}\right)^{m}\right)[p]\right) \\
& \cong\left(G^{*}[p] / G[p]\right) \oplus\left(\mathbb{Z}_{p}\right)^{m} .
\end{aligned}
$$

We will show that $\left(G^{*}[p] / G[p]\right)$ is the trivial group by showing that if $g \in G^{*}$, $p g=0$, then $g \in G$. Indeed, write $g=g^{\prime}+\sum_{b \in \mathcal{B}} y_{b} a_{b}$ with $g^{\prime} \in G$. Then

$$
0=p g=p g^{\prime}+\sum_{b \in \mathcal{B}} p y_{b} a_{b}=p g^{\prime}+\sum_{b \in \mathcal{B}} y_{b} b
$$

Since $0 \in p G$ has a unique representation (by Lemma 5.2) $0=0+\sum_{b \in \mathcal{B}} 0 b$, we get that $y_{b}=0$ for each $b \in \mathcal{B}$, and so $g=g^{\prime} \in G$.

By the previous lemma, we can recover $m$ from $\mathfrak{H}(G, m)$. We have

$$
p \mathfrak{H}(G, m)=p G^{*} \oplus p\left(\mathbb{Z}_{p}\right)^{m} \cong p G^{*}=G
$$

so that we can also recover $G$.

Thus, using Lemma 4.6, $\mathcal{M} \mapsto \mathfrak{H}(\mathfrak{G}(\mathcal{M}), \# \mathcal{M})$ gives a Borel reduction from $T_{p}$ to abelian $p$-groups. This completes the proof of Theorem 1.7 


\section{REFERENCES}

[AK00] Chris J. Ash and Julia F. Knight. Computable structures and the hyperarithmetical hierarchy, volume 144 of Studies in Logic and the Foundations of Mathematics. NorthHolland Publishing Co., Amsterdam, 2000.

$\left[\mathrm{FKM}^{+} 11\right]$ E. Fokina, J. F. Knight, A. Melnikov, S. M. Quinn, and C. Safranski. Classes of Ulm type and coding rank-homogeneous trees in other structures. J. Symbolic Logic, 76(3):846-869, 2011.

[FS89] Harvey Friedman and Lee Stanley. A Borel reducibility theory for classes of countable structures. J. Symbolic Logic, 54(3):894-914, 1989.

[Fuc70] László Fuchs. Infinite abelian groups. Vol. I. Pure and Applied Mathematics, Vol. 36. Academic Press, New York-London, 1970.

[HTIK] Matthew Harrison-Trainor, Gregory Igusa, and Julia F. Knight. Some new computable structures of high rank. Preprint.

[HTMM] Matthew Harrison-Trainor, Russell Miller, and Antonio Montalbán. Borel functors and infinitary interpretations. Preprint.

[HTMMM] Matthew Harrison-Trainor, Alexander Melnikov, Russell Miller, and Antonio Montalbán. Computable functors and effective interpretability. To appear in the Journal of Symbolic Logic.

[Mon] Antonio Montalbán. Computability theoretic classifications for classes of structures. To appear in the Proccedings of the ICM 2014.

[Mon15] Antonio Montalbán. A robuster Scott rank. Proc. Amer. Math. Soc., 143(12):54275436, 2015.

[Nad74] Mark Nadel. Scott sentences and admissible sets. Ann. Math. Logic, 7:267-294, 1974.

[Sco65] Dana Scott. Logic with denumerably long formulas and finite strings of quantifiers. In Theory of Models (Proc. 1963 Internat. Sympos. Berkeley), pages 329-341. NorthHolland, Amsterdam, 1965.

[Ulm33] Helmut Ulm. Zur Theorie der abzählbar-unendlichen Abelschen Gruppen. Math. Ann., 107(1):774-803, 1933.

[URL] Douglas Ulrich, Richard Rast, and Michael C. Laskowski. Borel complexity and potential canonical scott sentences. Preprint.

Group in Logic and the Methodology of Science, University of California, Berkeley, USA

E-mail address: matthew.h-t@berkeley.edu

$U R L:$ www.math.berkeley.edu/ mattht 